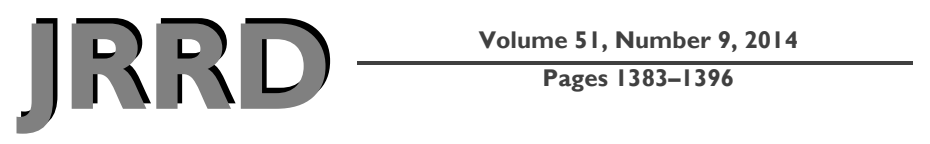

\title{
Development of telerehabilitation application with designated consultation categories
}

\author{
Kok Kiong Tan, PhD; ${ }^{1}$ Arun Shankar Narayanan; ${ }^{1 *}$ Gerald Choon-Huat Koh, PhD; ${ }^{2}$ Ko Ko Htet Kyaw; ${ }^{1}$ Helen \\ M. Hoenig, MD, MPH $^{3}$ \\ ${ }^{1}$ Department of Electrical and Computer Engineering and ${ }^{2}$ Saw Swee Hock School of Public Health, National Univer- \\ sity of Singapore, Singapore; ${ }^{3}$ Division of Geriatrics, Department of Medicine, Duke University Medical Center, Dur- \\ ham, NC
}

\begin{abstract}
Telerehabilitation (TR) is getting ever more popular because it is effective in bringing rehabilitation services to rural populations by means of audiovisual systems and its initial implementation studies presented encouraging results. TR is proven to be helpful, with benefits in terms of reduced travel time, cost, and availability of specialists' support in local communities. However, TR systems that are usable under low-bandwidth network environments are rare. This article introduces the development of a TR system with customized consultation categories for users to choose from, depending on requirements. Each category, with its preset parameter values, is discussed in detail by demonstrating relevant rehabilitation exercises. A novel bandwidth adaptation algorithm is also presented for optimal utilization of the dynamic network conditions, which ensures the system functionality even under narrow-bandwidth environments. Experiment results show that the system is able to perform effectively in each consultation category while the rehabilitation exercises are being performed. The proposed algorithm is also verified for its ability to adapt the content quality and effectively utilize the network under constrained conditions. A survey conducted on the video quality of the system under low-bandwidth conditions shows encouraging results for a large-scale deployment of the application.
\end{abstract}

Key words: consultation categories, low bandwidth, physiotherapy, rehabilitation, telehealth, telemedicine, telerehabilitation, TR application, TR system, videoconferencing.

\section{INTRODUCTION}

Telerehabilitation (TR) is an important subdiscipline of telemedicine in which modern telecommunication and information technology are used to deliver rehabilitation services over a distance. This is achieved by the use of technologies including audio, video, and virtual reality transmitted over the Internet. Comparison with traditional, in-person rehabilitation services reveals that costs can be reduced while maintaining or even improving overall effectiveness with appropriate use of TR [1]. Schein et al.'s study also reveals that TR is especially helpful for people by bringing advantages in terms of (1) decreased travel between the rural communities and specialized urban health centers, (2) availability of specialized clinical support in local communities, (3) indirect educational benefits for remote clinicians who

\footnotetext{
Abbreviations: $\mathrm{CQ}=$ compression quality, $\mathrm{FM}=$ fine motor, $\mathrm{fps}=$ frames per second, $\mathrm{GM}=$ gross motor, $\mathrm{PT} / \mathrm{OT}=$ physical therapist/occupational therapist, RBW = receiving bandwidth, SBW = sending bandwidth, TR = telerehabilitation.

*Address all correspondence to Arun Shankar Narayanan, National University of Singapore-Electrical and Computer Engineering, Blk E4A \#03-04 Engineering Drive 3 117576, Singapore; +65-8198-4744; fax: +65-6777-3117.

Email: a0035708@u.nus.edu

http://dx.doi.org/10.1682/JRRD.2014.02.0052
} 
participate in teleconsultations, and (4) alleviation of feeling of isolation for rural clinicians [2].

According to the latest report from the International Telecommunication Union, 41 percent of the world's households are connected to the Internet and 50 percent of them are in developing countries. However, the report notes that high-speed access to the Internet is still limited in developing countries due to the cost factor, with fixedbroadband service accounting for 30.1 percent of average monthly incomes. For this reason, in the majority of developing nations, Internet speed can be approximately 256 kbps or less [3]. This means that for a TR system to work satisfactorily in such environments, it should be able to adapt its content quality to the Internet speed available. Even in developed countries, high and fixed bandwidths come at a premium and the group of people for whom TR would be most useful are often the least likely to have premium Internet access. For example, according to the latest statistics from the Infocomm Development Authority of Singapore, a decent Internet speed connection (more than $50 \mathrm{Mbps}$ ) costs on average $\$ 50$ or more a month. The presented data also reveal that for Internet networks with a maximum speed of $50 \mathrm{Mbps}$ or below, the uploading speed is in terms of a few hundred kbps, which is well below the necessary requirement for a high data rate communication [4-5].

A typical TR scenario includes motion assessments related to the gait, fine motor (FM) skills, and gross motor (GM) skills of a patient. FM skills refer to activities related to fingers, wrists, and eye-hand coordination, all of which involve smaller muscle movements [6]. GM skills refer to activities such as the process of sitting, walking, running, and jumping, during which the major body muscles are in motion [7]. The speed at which these various exercises are carried out varies; FM exercises involve fast movements, whereas GM exercises are slower. Thus, the video quality requirements for each of these situations will be different. Some need a high resolution, whereas some others need a high frame rate. By optimizing video parameters such as frame rate and resolution, assessment performance in each of these cases can be maximized in a limited bandwidth environment.

A study conducted by Hoenig et al. [8] examined the effect of differing network environments on measurement accuracy when examining physical function by using standard, off-the-shelf videoconferencing technology for telemedicine. The study provided promising results for assessing FM function when the bandwidth was high (768 kbps), but the accuracy dropped significantly when the same system operated at lower bandwidths, including 384 and 64 kbps. Moreover, accuracy was suboptimal at all bandwidths for assessing GM function (e.g., gait) and it only rose to acceptable levels relative to in-person assessment with use of slow-motion review of a high quality videorecording. The authors suggested that differing types of motor function have differing technological needs (e.g., frame rate, resolution, stereoscopic image) for optimal assessment via telehealth. The article concluded that improved technology and infrastructure were needed to better meet the physical therapist's (PT's)/occupational therapist's (OT's) clinical requirement for telehealth.

With the insights gained from the Hoenig et al. study [8], in this article we introduce the concept of having different categories for televideo transmission of a TR session, termed "TR consultation categories," particularly for PT/OT TR consultations, where users can select a particular category with a predefined parameter setting that ensures maximum transmission of clinically useful data in limited-bandwidth environments. The techniques used in this article are based on another article by the same authors [9] that discussed different methods of reducing the data size while conducting a telemedicine session over a narrow-bandwidth network, with a new approach formulated for the bandwidth adaptation as explained in the "System Overview" section. This article further discusses the effectiveness of having appropriate TR consultation categories for different TR sessions, followed by the necessary evaluation to validate the claims.

\section{METHODS}

\section{Literature Review}

The acceptability of telecommunication technology use in rehabilitation practice has been substantiated in many studies. Finkelstein et al. conducted a pilot study on home-based physical TR and the results were promising, with an acceptance rate of more than 80 percent from the patients [10]. An early study by Dick et al. reported that 76 percent of the patients who participated in the telemedicine assessment were satisfied with the system [11]. Regarding the technical acceptability of information obtained via TR, an overall agreement of 92 percent was reached between the video-based assessment and the face-to-face assessment in a study conducted by Rintala 
et al. [12]. Sanford and Hoenig compared an individualized, comprehensive multifactorial intervention aimed at improving a patient's mobility provided either by a therapist in person in the patient's home or through use of TR and found that the two methods found a similar number of problems, recommended a similar number of interventions, and adopted the interventions at a similar rate with either mode of service delivery [13].

A study conducted by Schein et al. on the interrater reliability between in-person and TR assessment of Functioning Everyday with a Wheelchair-Capacity demonstrated excellent results, with an interclass correlation coefficient of 0.91, although the system was tested under high-bandwidth conditions [14]. In a separate study, Schein et al. again compared TR sessions on wheeled mobility and seating assessments with in-person assessment scenarios and the results indicated that the TR sessions were equally effective as the "gold standard" inperson assessments [2].

Sanford et al. conducted clinical trials in a study to evaluate the effectiveness of PT/OT sessions through televideo technology for follow-up visits. The study reported that potential use of televideo sessions was encouraging for meeting the needs of in-home PTs/OTs [15]. In a study on remote assessment of low back pain, Truter et al. also addressed the practicality of TR, in particular by PTs in rural clinical settings. The participant satisfaction was good overall, with acceptable performance in assessments, strong correlation with in-person results, and high reliability scores [16]. This emerging evidence is encouraging for the use of TR as a supplement to or even in place of face-to-face sessions.

Although there are existing systems for telemedicine purposes, they are not specifically designed to perform well in low-bandwidth environments. Parmanto et al. developed a versatile TR system [17], used in some of the just-mentioned studies, which supports audiovisual data exchange. However, the system was designed for broadband networks, and thus, it is not suitable for narrowband communication when the bandwidth drops to low levels. Panayides et al. developed an open source telemedicine platform for wireless medical video communication [18]. Similarly, this solution did not specifically address the issue of communication in lowbandwidth networks.

From the literature survey conducted, no prior work was found on development of solutions that are adaptable to the stringent requirements of low-bandwidth networks in terms of multiple data parameters and at the same time, user options for selecting a consultation category based on specific requirements of a telemedicine session. In this article, a TR system for PT/OT use in particular was designed and developed in which the user was able to select the consultation category based on the assessment to be performed. Each consultation category was designed to give priority to different video parameters (e.g., frame rate, compression quality [CQ], color), depending on the application requirements as mentioned earlier. Evaluations were done while the system was operated in a limited-bandwidth environment, and conclusions were drawn on the effectiveness of such a system.

\section{System Overview}

\section{System Architecture}

The system is simple on the PT's/OT's end, with a normal webcam and microphone and the software running on a Windows 7 PC (Microsoft Corporation; Redmond, Washington). On the patient's end, the webcam is replaced with Microsoft's Kinect sensor in order to make use of some of its extra functionalities, such as depth sensing, as described by Arun and Tan [9]. The software was developed on Microsoft Visual Studio using C\# programming language. The overall system runs as a clientserver architecture whereby a central server is the connection point for both clients, the patient and the PT/OT. Audio, video, and other data from either client sides are transferred through dedicated ports at the fixed server at all times.

\section{Novel Bandwidth Adaptation Method}

The bandwidth detection algorithm by Arun and Tan [9] was based on sending a fixed-size packet from the sender and calculating the available bandwidth based on the time difference between the sending time and the acknowledgment return time from the receiver. This method, however, was not ideal for detecting the speed in low-bandwidth networks due to the additional payload caused by the fixed-size packet. The fundamental assumption was that processing and other related delays are negligible compared with the actual return time of the message packet. This assumption holds true only if the packet size is large, in terms of hundreds of kilobytes; otherwise, the detected bandwidth value will be inconsistent. The algorithm by Arun and Tan employed a message packet-sized $200 \mathrm{kB}$ sent in every $2 \mathrm{~min}$, and this 
induced additional burden on the already limited network bandwidth. Another limitation of this algorithm was that it was not quick enough to identify the changes in bandwidth and adapt the video quality accordingly.

To improve on the aforementioned limitations, a new method is introduced in this article to accurately detect the available bandwidth; the algorithm flowchart is shown in Figure 1. A timer is set up to trigger every $5 \mathrm{~s}$ to measure the data rate, for the next $2 \mathrm{~s}$ from the trigger, at the sending client (SBW1) as well as the data rate at the receiving client (RBW1). If SBW1 is found to be equal to RBW1, this implies further availability of bandwidth, and thus, the video parameter is increased at the sending side in order to maximize the bandwidth utiliza- tion. If the selected consultation category is of frame rate priority, CQ will be increased by factors of 5 until a maximum value of 80 percent is reached. Once the CQ value hits 80 percent and there is still bandwidth available, frame rate will start to increase up to the maximum value of 30. Similarly, for the resolution priority classes, frame rate will be increased by 1 until the maximum value of 30 frames per second (fps) is reached. Once the maximum frame rate is reached and bandwidth is still available, CQ will be increased until a maximum value of 80 percent. This is done to ensure that the system is able to utilize the available bandwidth to the maximum extent possible.

If SBW1 is more than RBW1, which implies that the maximum available bandwidth is reached at the receiver

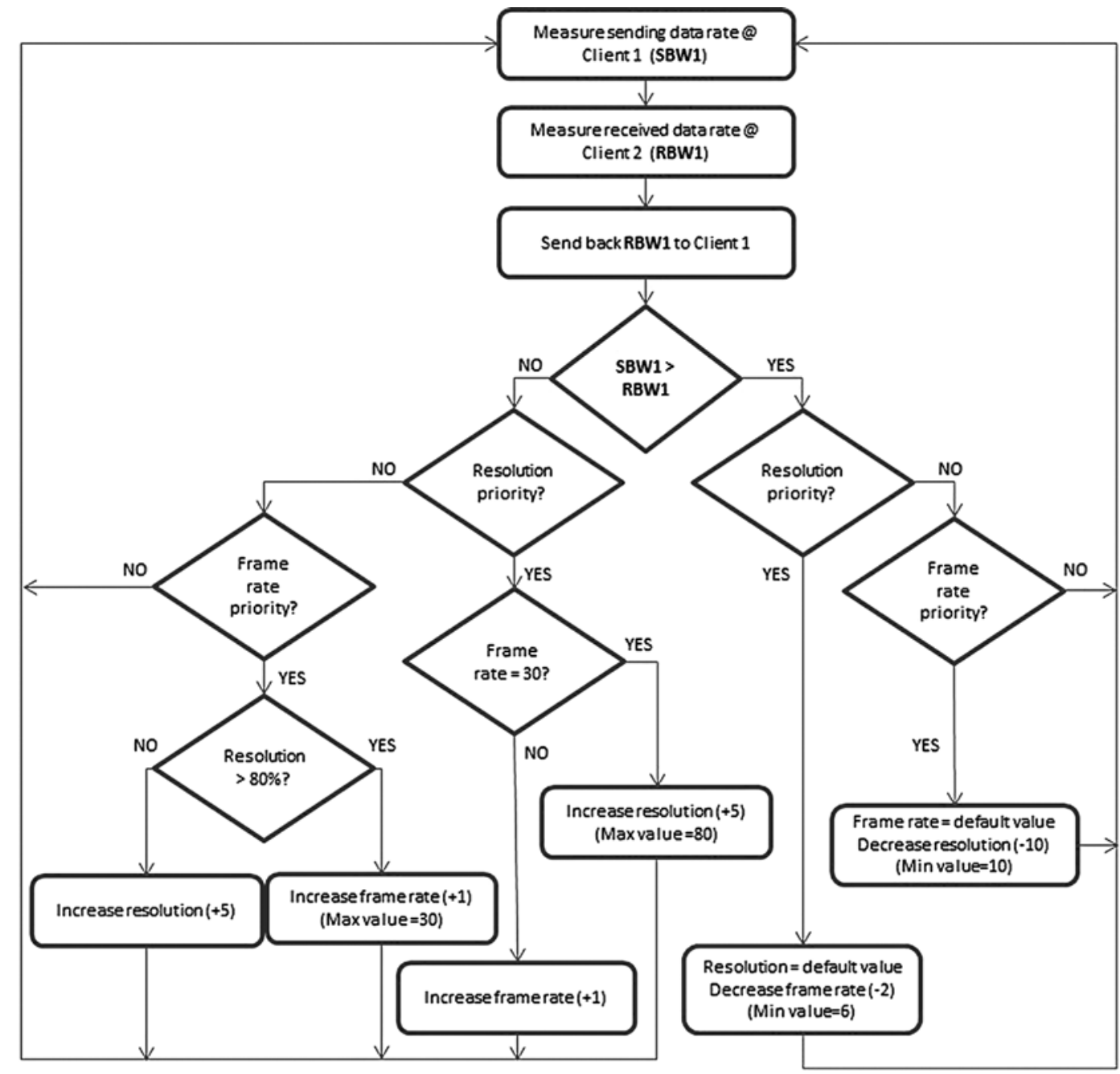

Figure 1.

Bandwidth detection algorithm at client 1. Max $=$ maximum, Min = minimum. 
end, the corresponding parameters will be reduced according to the selected consultation category until the minimum value is reached. The minimum value for CQ is set at 10 for frame rate priority classes, whereas minimum frame rate is set at 6 for resolution priority classes. These values are chosen as minimum requirements based on numerous trial runs because they were found to be the minimum values for ensuring a meaningful consultation session. The parameter increments/decrements, as shown in Figure 1, were chosen roughly based on an additiveincrease/multiplicative-decrease algorithm [19]. The whole process repeats every $5 \mathrm{~s}$, and thus, the bandwidth adaptation is much faster than the earlier method discussed by Arun and Tan [9]. This interval, i.e., $5 \mathrm{~s}$, is an empirical choice to strike a balance between adaptation speed and program resources. If too long a sampling value is chosen for this purpose, the adaptation will be slow; similarly, too short a value will cause the program to be constantly measuring the data rate, which is also not desirable to the system.

\section{Telerehabilitation Consultation Categories}

As mentioned earlier, different consultation categories were provided for the user to choose from depending on the assessment requirements. Leveraging the techniques mentioned by Arun and Tan [9], five categories were identified for classification. The parameters varying in each category are (1) frame rate, (2) resolution or CQ, (3) color, (4) manual or automatic cropping, and (5) spatial data extraction using Kinect sensor. Although the default values are set for each parameter in each category, the users have the freedom to change some of them according to requirements. Details of the five consultation categories and their default parameter settings are explained as follows.

Fine motor. FM in a human body refers to the smaller muscles (e.g., hands, wrists, fingers). Activities relating to FM skills include picking and sorting small objects using tongs or tweezers and holding and using feeding utensils. In order to make an accurate assessment during a typical rehabilitation session, the PT/OT might need to focus on the quality of the movement to identify problems with incoordination or trembling. If this is the priority, in a low-bandwidth TR session, priority would need to be set to frame rate so that all the FM movements are able to be seen. Other parameters such as video color and resolution may not be as critical in this particular situation and, thus, given less weightage in consideration.
At other times, it may be important to be able to detect problems related to the interface of the hand with the environmental task parameters (e.g., fumbling with buttons, problems manipulating a tool or a utensil properly), in which case a highly detailed view may be important and parameters such as video resolution and/or color would need to be prioritized in a low-bandwidth session. Moreover, it may be necessary to repeat tasks so that they can be viewed with a high frame rate to assess the quality of the movement and again with high resolution or video color to assess the function of the hand and fingers in particular aspects of the task being performed. To cater to such circumstances, the system is designed to allow the user to switch the priority from frame rate to resolution by manual intervention, with the default system setting being the frame rate priority scheme.

Spatial data extraction, as mentioned by Tan et al. [20], is optional in this category, with the user having the ability to activate it when needed. Figure 2 shows a sample screenshot of the application at the patient's side when the FM category is chosen and color information from the patient's end is activated. The frame rate is set at minimum 15 fps by default while resolution is varied dynamically up to 80 percent if the bandwidth permits, with a minimum guaranteed CQ value of 10 percent. Once the maximum value of CQ is reached and the system detects further available bandwidth, the frame rate will be increased in order to make full use of the network. Cropping is not activated in this case because the relative position of body parts with respect to the background may be useful for certain assessment purposes.

Gross motor. GM skills refer to the movement of major muscles of human body during exercises such as walking, weight lifting, and throwing a ball. Compared with FM exercises, GM skills are slower in pace, but assessing continuity of movement nonetheless may require a high frame rate (e.g., concern about intermittent loss of balance while walking, determining the presence of abnormalities in gait cadence). However, sometimes clinical concerns arise about subtle movement abnormalities (e.g., normal vs abnormal amount of sway, step width), in which case relative priority may need to be placed on resolution over frame rate. Thus, as with FM movements, it is important the clinician be able to utilize the optimal technological parameters and be able to alter the parameters during the clinical visit as the evaluation progresses.

The resolution priority case will be activated, by default, in this category by setting the minimum CQ of 
(a)

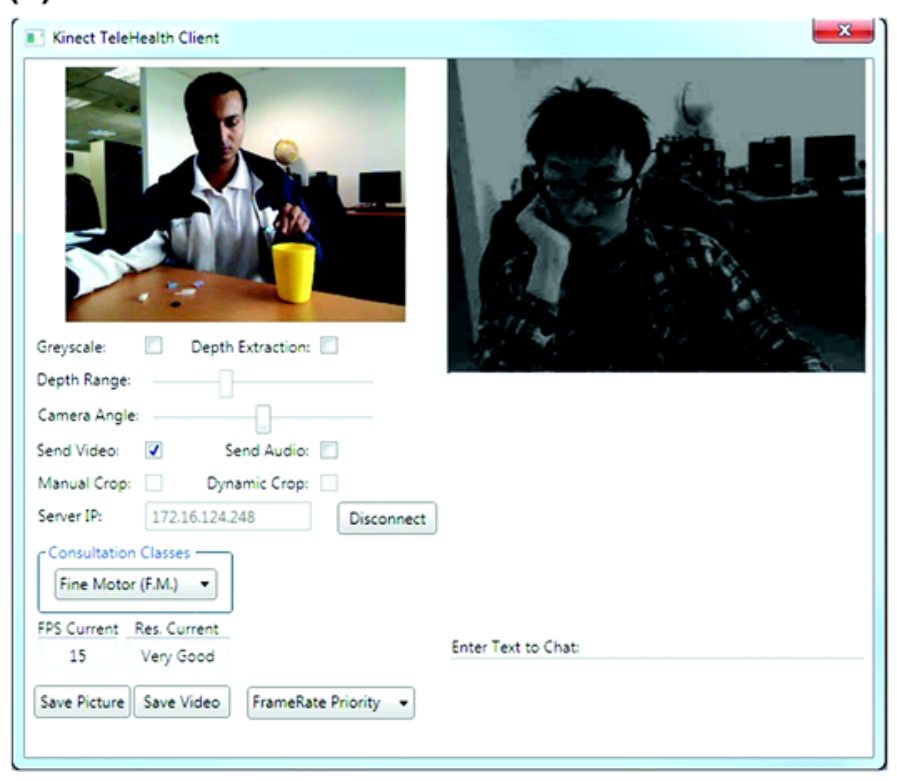

(b)

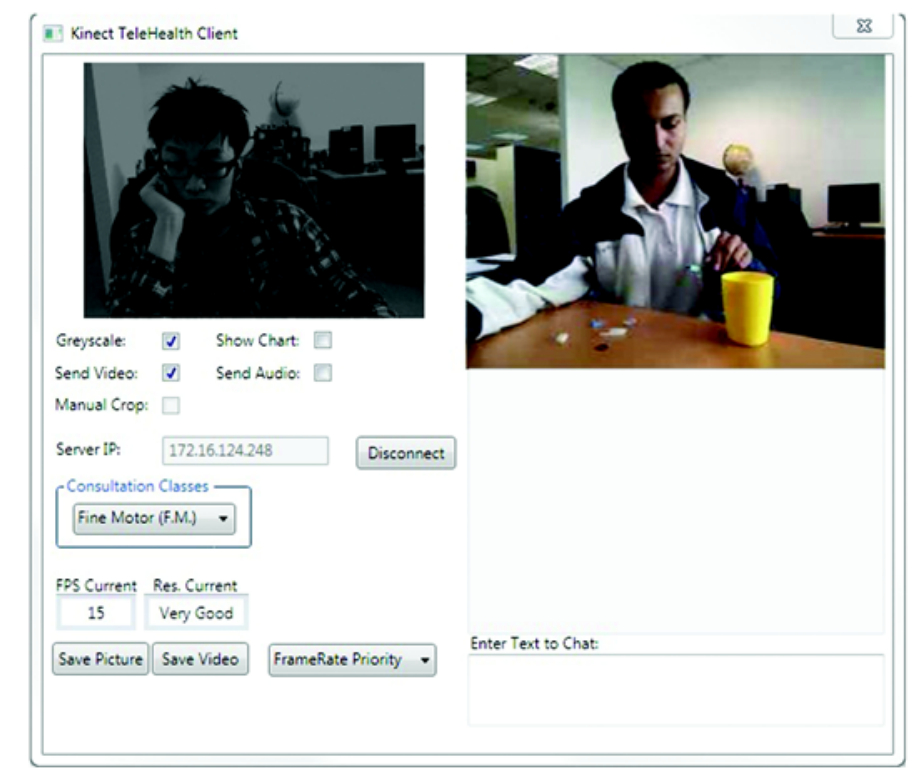

Figure 2.

Sample screens with fine motor category selected at (a) patient's and (b) health professional's end.

30 percent to be maintained at all times, thus ensuring a minimum image resolution, while dynamically changing the frame rate in accordance with the available network bandwidth every $5 \mathrm{~s}$. Based on the trial runs, the minimum value for frame rate was set as 6 while the maximum value was set at the camera limit of 30 . Once the maximum frame rate value is reached and available bandwidth is still more than the data rate, CQ value will increase in order to increase the consultation quality by maximizing the bandwidth utilization. However, to cater to the other scenarios that require high frame rate, as mentioned earlier, the user will have the option to switch the priority from resolution to frame rate in this category as well.

Finally, some GM and FM tasks require the ability to discern movement in 3 dimensions and/or discern the interface of the patient with the environment. Examples of the former might be step continuity while turning or evaluating patient performance during contextually embedded dynamic tasks such as climbing stairs, transferring to the toilet or wheelchair, reaching, and manipulating items during food preparation. Examples of the latter include assessing foot clearance while walking and fumbling while manipulating objects. Since the background information may be needed to monitor the relative position of body parts and/or problems interfacing with the environment, cropping is deactivated by default in this category. As in the previous case, spatial data extraction and color are set as optional parameters. Figure 3 shows a screenshot of the application when the GM category is selected with the spatial data extraction feature activated. The image is shown to be tracking the arm movement while the patient carries out a weight lifting exercise. The angle formed between the joints of shoulder, elbow, and hand is measured and displayed together with the hand velocity data by using the spatial data extraction feature.

Fine motor in isolation. This category is a variant of the FM category discussed earlier, but catered to different FM exercises. Other examples of FM exercises include finger tapping and handwriting. While carrying out such exercises and monitoring through video, only a part of the screen is of interest to the physician. Thus, in such cases, the unwanted areas of the screen can be omitted from being sent over the network by employing the cropping feature. In order to select the region of interest, the user just has to draw a rectangle across the desired part in the image screen. This feature helps to reduce the data payload further and, thus, ensures a smoother TR session even in limited network bandwidth environments. 


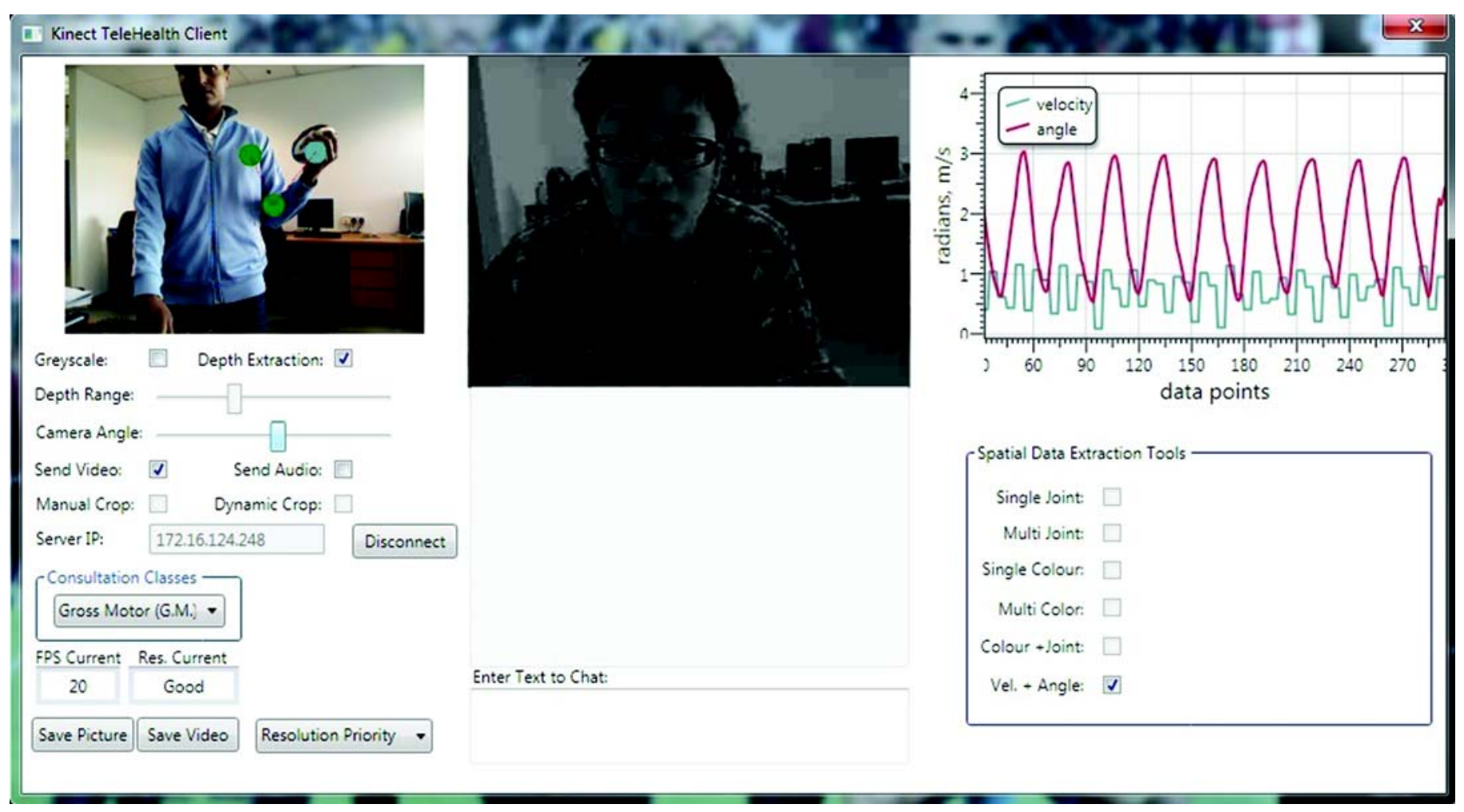

Figure 3.

Sample screen with gross motor category selected and spatial data activated.

This category is similar to as described in the "Fine Motor" section, except that the tasks can be performed in isolation by the cropping mechanism. The user will also have the option to turn on color, if needed, because the cropping mechanism helps to make room for more data to be accommodated. Other than manually cropping off the area of interest in the screen, the user can activate dynamic cropping as well, provided a Kinect camera is used instead of a normal webcam. It works by masking out the objects farther than a specified distance from the camera with the help of Kinect's depth sensor. Since data size can be significantly reduced by the cropping mechanism, the minimum frame rate to maintain in this category was chosen as 20, while the resolution remained dynamic in accordance with the available bandwidth of the network with the same range of values as mentioned in the "Fine Motor" section. Spatial data extraction and color are again optional depending on the user's preference. Figure 4 shows a screenshot of the application in this category with manual cropping activated. As in the earlier case, the user will have the option to switch priority from frame rate to resolution in this category as well.

Gross motor in isolation. This category is a variant of that in the "Gross Motor" section, in which GM tasks are assessed, but caters to different exercises. This is based on resolution priority case as well, with frame rates varying according to the available network speed every $5 \mathrm{~s}$. The CQ value to maintain in this category was chosen to be at a higher rate of 40 percent because of the data size reduction made possible by the use of the cropping feature, with the same range of frame rate values as in the "Gross Motor" section. Hence, this category is useful for situations in which only the patient's body is the area of interest and the surrounding environment can be ignored. The resulting data reduction allows the video to be transmitted with color data. Similar to previous categories, a spatial data extraction feature is optional and can be activated when needed. Again, the priority scheme can be switched from resolution to frame rate upon user intervention. Figure 5 shows a screenshot of this category 


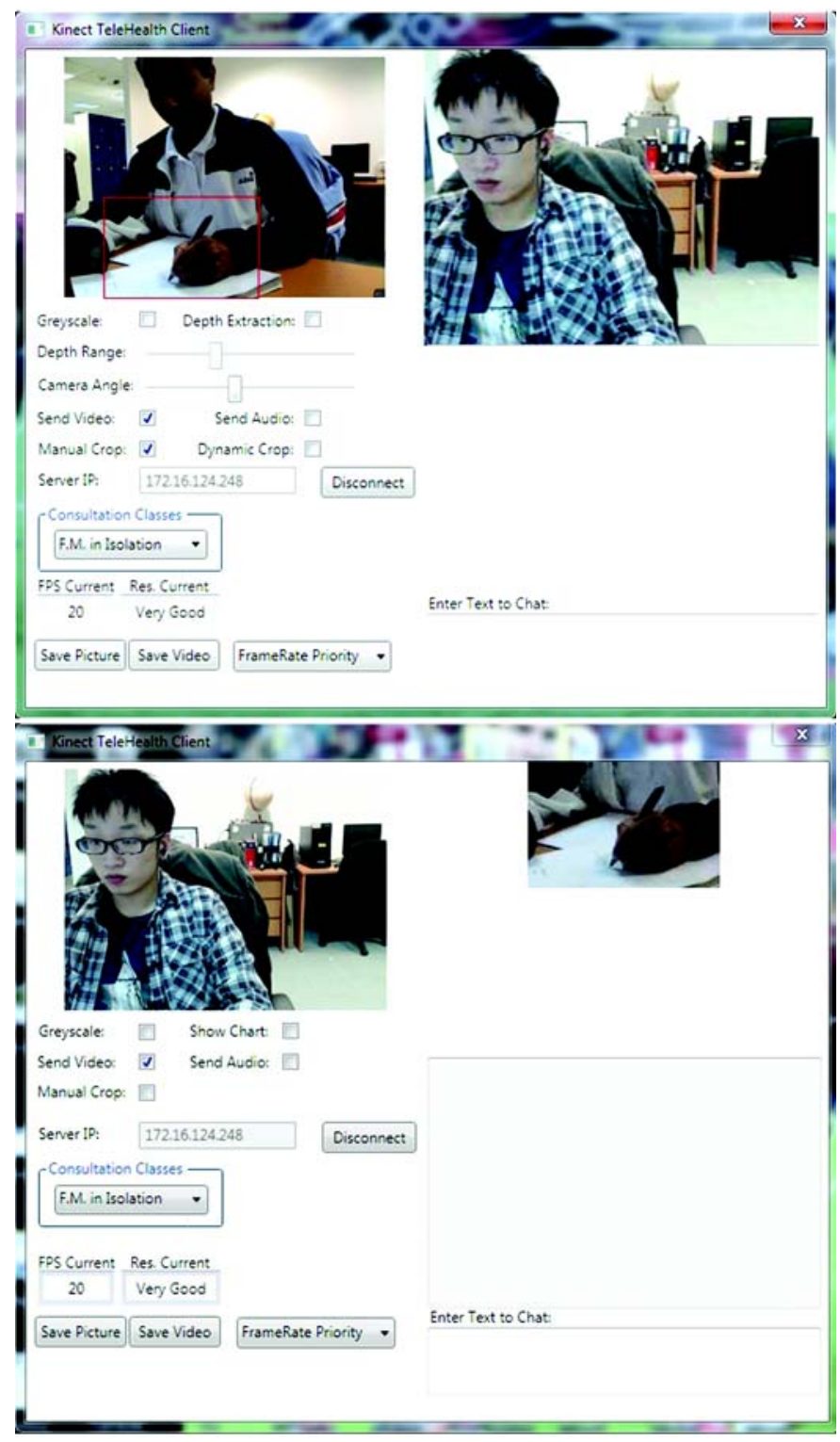

Figure 4.

Sample screens with fine motor in isolation category selected and manual cropping enabled.

with dynamic cropping turned on while assessing a patient performing a walking exercise.

Manual category. Although there are four categories with preset parameters to choose from, some assessments may require different parameter settings, e.g., different frame rate or resolution values. To cater to such cases, a manual category was constructed in which the user can adjust the frame rate or the resolution quality

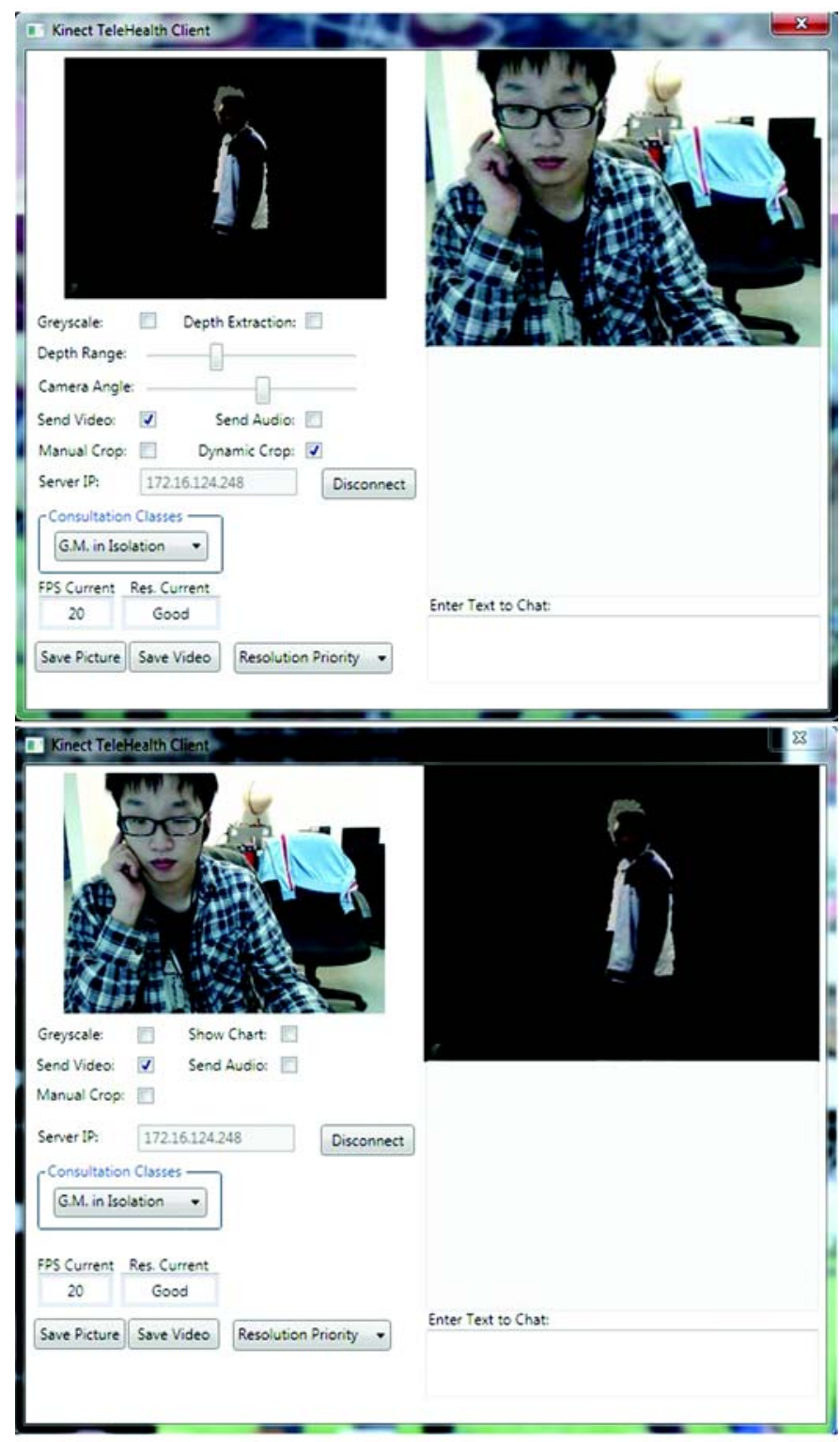

Figure 5.

Sample screens with gross motor in isolation category selected and dynamic cropping enabled.

freely. Although the system does not alter either the frame rate or resolution dynamically in this category, the bandwidth detection algorithm is still running and recommended values for both parameters are shown to the user. However, the selection of these parameter values is left to the user's discretion, and consequently, the system can not guarantee a smooth performance if higher than acceptable values are chosen in a limited-bandwidth network environment. 
All the parameters available in the system are made optional in this category, with the user having the freedom to activate them when the need arises. Figure 6 shows the system with manual category selected.

Table 1 summarizes the default settings of all parameters in each of the five consultation categories explained previously. As mentioned earlier, parameters such as color, cropping, and spatial data extraction can be activated if the need arises during the consultation session and, thus, have been set as optional.

\section{RESULTS AND DISCUSSION: SYSTEM EVALUATION}

\section{Minimum Bandwidth Requirements}

Experiments were conducted in order to determine the bare minimum requirement for the system to function satisfactorily in frame rate priority and resolution priority cases. The manual consultation category was chosen to run the experiment, and frame rate and CQ values were manu- ally changed, while color information as well as spatial data extraction features were disabled. The experiments were conducted in two sets, the first set using the Kinect camera/webcam with $640 \times 480$ frame resolution (at the patient's end) and the second set using a built-in laptop webcam with $320 \times 240$ frame resolution (at the PT's/OT's end). Although the difference in frame resolution was exactly half in the built-in webcam case as compared with the Kinect camera, the bandwidth utilization did not follow this scale exactly, probably because of compression algorithm dynamics.

Table 2 shows the measured bandwidth while the system was in operation under the aforementioned conditions. From the data, it is clear that the system would function smoothly even when the available bandwidth dropped below $128 \mathrm{kbps}$, if operating within its minimum parameter settings. While using the system with a built-in webcam in a laptop, it can function in resolution priority categories even when the bandwidth availability drops below $50 \mathrm{kbps}$. This is one of the most important features

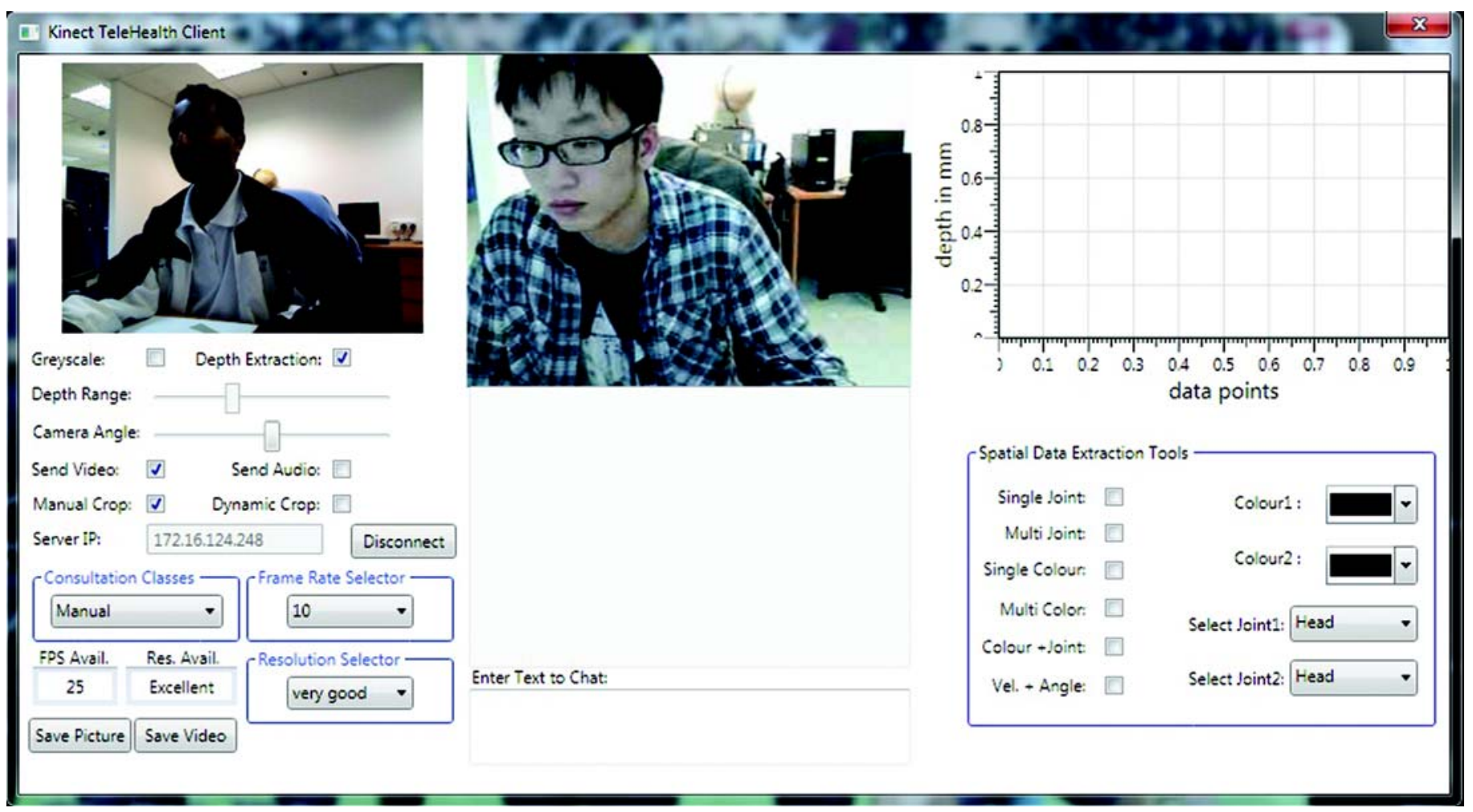

Figure 6.

Sample screen with manual category selected. 
JRRD, Volume 51, Number 9, 2014

Table 1.

Overview of consultation categories in telerehabilitation system.

\begin{tabular}{llllll}
\hline \multicolumn{1}{c}{ Category } & \multicolumn{1}{c}{ Frame Rate } & $\begin{array}{c}\text { Resolution } \\
\text { (Compression Quality) }\end{array}$ & Color & Cropping & $\begin{array}{c}\text { Spatial Data } \\
\text { Extraction }\end{array}$ \\
\hline Fine Motor & 15 (minimum) & Varying (10\%-80\%) & Optional & Off & Optional \\
Gross Motor & Varying (6-30 fps) & $30 \%$ (minimum) & Optional & Off & Optional \\
Fine Motor in Isolation & 20 (minimum) & Varying (10\%-80\%) & Optional & Optional & Optional \\
Gross Motor in Isolation & Varying (6-30 fps) & $40 \%$ (minimum) & Optional & Optional & Optional \\
Manual & User defined & User defined & Optional & Optional & Optional \\
fps = frames per second. & & & & &
\end{tabular}

fps $=$ frames per second

Table 2.

Bandwidth measured with system operated at minimum parameter settings.

\begin{tabular}{llccccc}
\hline \multicolumn{1}{c}{ Camera } & Priority & $\begin{array}{c}\text { Frame Rate } \\
\text { (fps) }\end{array}$ & $\begin{array}{c}\text { Resolution } \\
\text { (Compression Quality, \%) }\end{array}$ & $\begin{array}{c}\text { Color } \\
\text { Spatial Data } \\
\text { Extraction }\end{array}$ & $\begin{array}{c}\text { Bandwidth Used } \\
\text { (kbps) }\end{array}$ \\
\hline Kinect/Webcam & Frame Rate & 15 & 10 & Off & Off & 100 \\
& Resolution & 6 & 30 & Off & Off & 60 \\
Built-in Webcam & Frame Rate & 15 & 10 & Off & Off & 70 \\
& Resolution & 6 & 30 & Off & Off & 40 \\
\hline fps = frames per second. & & & & &
\end{tabular}

of this system and enables the teleconsultation session to be carried out in bandwidth-scarce environments.

\section{Efficiency of Bandwidth Adaptation Algorithm}

Ability of the system to adapt itself to the dynamics of available network bandwidth is key to the effectiveness of its implementation in bandwidth-scarce environments such as rural areas. This test was performed to measure the effectiveness of this system's adaptability while the bandwidth available fluctuated. A third-party software, NetLimiter [21], was used for simulating such a scenario. The bandwidth was increased from an initial value of $32 \mathrm{kBps}$ (256 kbps) to $44 \mathrm{kBps}$ (352 kbps) in short steps of $4 \mathrm{kBps}$ and then reduced to $28 \mathrm{kBps}$ (224 kbps) in a

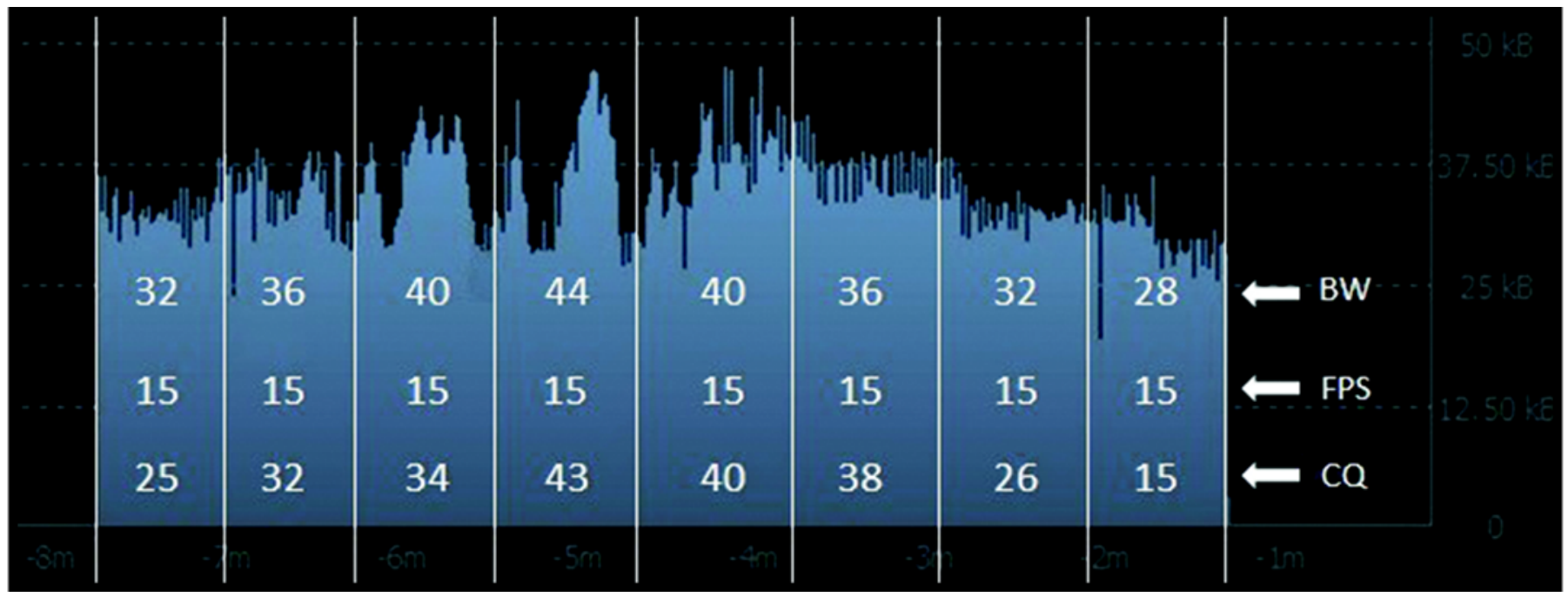

Figure 7.

Efficiency of bandwidth (BW) adaptation algorithm of the system under fine motor category. CQ = compression quality, FPS = frames per second. 
similar fashion, and the data rate adaptation by the system was monitored. Figure 7 shows the chart with the data rate variation carried out by the software during this experiment while operating in the FM category. Figure 7 also displays the frame rate maintained as well as the CQ value, adapted to the available bandwidth. The horizontal axis shows the time in minutes and the vertical axis shows the data rate in kilobytes per second.

Similarly, Figure 8 shows the bandwidth adaptation operated in the GM category. The minimum CQ value was maintained throughout the experiment and the frame rate varied as shown in the figure. Both experiments were repeated three times to obtain an average value of parameter settings during bandwidth adaptation.

It is clear from Figures $\mathbf{7}$ and $\mathbf{8}$ that the system is adapting its data rate to the available bandwidth by varying the corresponding parameters gradually, and it is able to respond to the dynamics of the network traffic (i.e., by steadily varying $C Q$ in the first case and frame rate in the other).

\section{Efficiency of Available Bandwidth Utilization}

This test was conducted to quantify the ability of the system to successfully measure and utilize the maximum available bandwidth and, thereby, ensure a good quality video consultation session at all times. Again, NetLimiter was used to simulate different bandwidth environments by restricting the data transfer rate at the server and the utilization was measured in each environment. Table 3 tabulates the percentage utilization of the available bandwidth in four different bandwidth scenarios. The first three columns show the system's bandwidth utilization in FM and GM, which employ the bandwidth adaptation algorithm, and the last two columns show the utilization if the system is operated without any bandwidth adaptation. The manual category is selected in the latter case, because bandwidth adaptation was not functioning in this category, with the parameter values fixed at a default setting in order to suit the slowest bandwidth scenario (choosing a higher parameter setting will cause system hang because of excessive data being transferred through a narrow network path).

Comparing the results in Table 3, in all the FM and GM scenarios the average utilization was found to be above 95 percent of the available bandwidth and hence ensuring that the system provided a good quality consultation session at all times; however, in the manual case, the bandwidth remained constant even when the available bandwidth increased, resulting in bandwidth underutilization.

\section{Video Quality Assessment Survey}

A survey was conducted to gather information on the video quality delivered in low-bandwidth networks while employing the data reduction techniques mentioned in this article. Two exercises were chosen, one involving FM

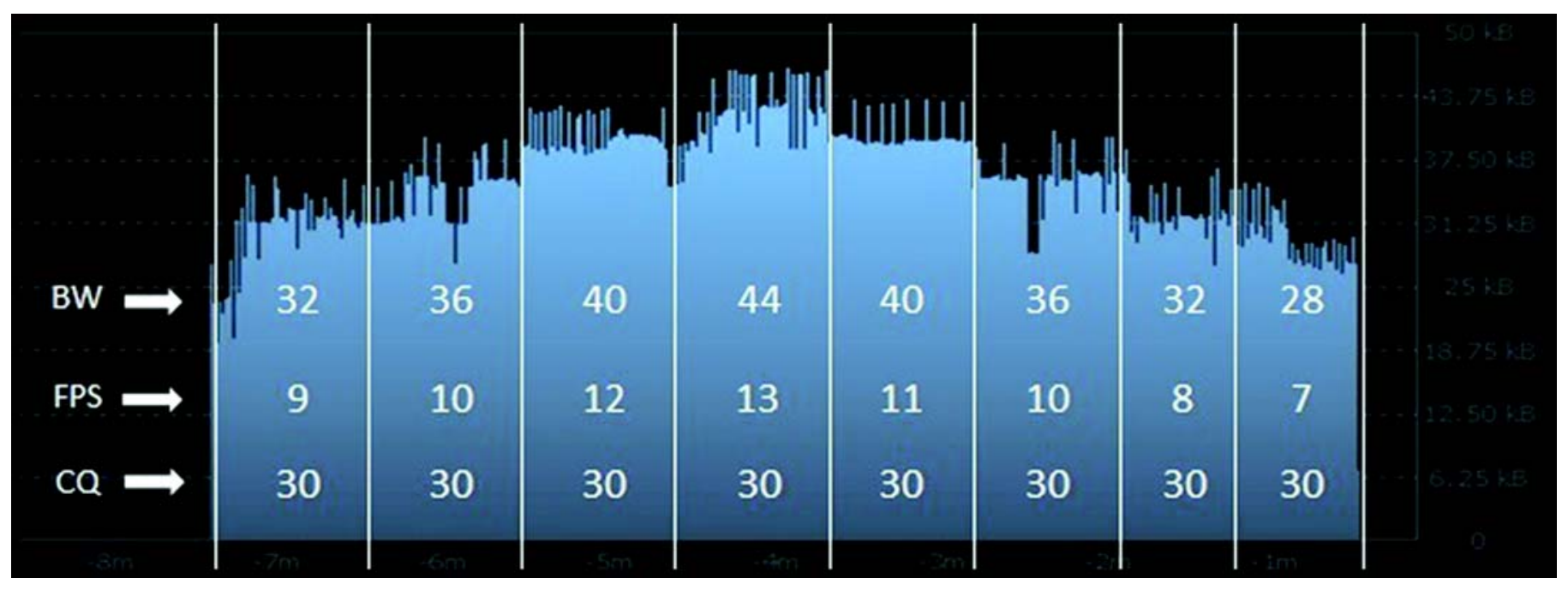

Figure 8.

Efficiency of bandwidth (BW) adaptation algorithm of the system under gross motor category. CQ = compression quality, FPS = frames per second. 
JRRD, Volume 51, Number 9, 2014

Table 3.

Bandwidth utilization by the system in different network environments.

\begin{tabular}{lcccccc}
\hline \multirow{2}{*}{$\begin{array}{c}\text { Available } \\
\text { Bandwidth (kbps) }\end{array}$} & \multicolumn{2}{c}{$\begin{array}{c}\text { Bandwidth Used by } \\
\text { System (kbps) }\end{array}$} & \multirow{2}{*}{$\begin{array}{c}\text { Average Use in Fine and } \\
\text { Gross Motor (\%) }\end{array}$} & \multicolumn{2}{c}{$\begin{array}{c}\text { Bandwidth Use with } \\
\text { No Adaptation }\end{array}$} \\
\cline { 2 - 3 } & Fine Motor & Gross Motor & & & kbps & \% \\
\hline 128 & 124 & 125 & 239 & 97 & 125 & 97 \\
256 & 251 & 378 & 96 & 124 & 48 \\
384 & 376 & 474 & 95 & 130 & 34 \\
512 & 503 & & 98 & 125 & 24 \\
\hline \hline
\end{tabular}

motion and the other GM motion, to provide quantitative measures of system performance. The first exercise was a finger-tap workout carried out by a coauthor serving as the subject. The subject was asked to perform the exercise by tapping the index finger on the table 10 times at around 2 taps/s, repeated over three sets. The exercise video was streamed across a low-bandwidth network of less than $200 \mathrm{kbps}$, first by using the adaptive bandwidth algorithm by selecting the FM in isolation category and then by using the manual category where no bandwidth adaptation strategy was employed. Similarly, a second exercise was transmitted across a low-bandwidth network. The subject was asked to perform an elbow flexion-extension motion repeated over three sets such that in each set, five full extensions of the elbow, were followed by a single half extension. The second exercise was initially run by selecting the GM category followed by the manual category.

The received video stream in all four cases was recorded and evaluated by independent testers, including certified therapists, care providers, and technicians who were trained on the use of the system. The recorded video files are available online. The testers provided their assessments via a questionnaire, and the results are shown in Table 4. The survey responses clearly indicate that by employing the bandwidth adaptation strategies as explained in this article, the system is indeed able to perform well under low-bandwidth environments. The movement smoothness and the image clarity were unani- mously assessed to be much better in the case of adaptive streaming, and thus, the developed system is favored for use in such constrained conditions.

\section{CONCLUSIONS}

The design and development of a TR system for PT/ OT consultation was discussed in this article. A new method for bandwidth adaptation was introduced, and different consultation categories were identified based on the different requirements of a TR session. Each consultation category was formed with varying parameter settings, such as frame rate, CQ, color information, cropping features, and spatial data extraction. The developed system was evaluated for the minimum bandwidth required to be used successfully in a teleconsultation, and it was shown to be able to operate even when the network speed dropped below 128 kbps. Efficiency of the bandwidth adaptation algorithm was tested and verified by abruptly changing the available network speed. The system was also tested for its efficiency in utilizing the maximum available bandwidth and was able to exploit more than 95 percent of the available speed. The evidence from the experimental results as well as the ground survey responses suggest that with the implementation of the developed system in a bandwidth-limited environment in scenarios such as rural areas in developing countries, it is

Table 4.

Survey response on recorded trial videos.

\begin{tabular}{|c|c|c|c|c|c|c|}
\hline \multirow{2}{*}{ Motion } & \multicolumn{2}{|c|}{ Average No. of Taps Seen } & \multicolumn{2}{|c|}{ Video Clarity* } & \multicolumn{2}{|c|}{ Movement Smoothness* } \\
\hline & Adaptive & Manual & Adaptive & Manual & Adaptive & Manual \\
\hline Finger-Tap Motion (Fine Motor) & $10($ true value $=10)$ & 7 (true value $=10)$ & 5.0 & 2.0 & 4.5 & 1.7 \\
\hline
\end{tabular}


possible to conduct smooth TR sessions with specialists who may be residing in the city or even in another part of the world. In due course, trials will be conducted in realworld clinical settings in rural areas where Internet connectivity is highly likely to be less than ideal.

\section{ACKNOWLEDGMENTS}

\section{Author Contributions:}

Study concept and design: K. K. Tan, A. S. Narayanan, H. M. Hoenig. Acquisition of data: A. S. Narayanan, K. K. H. Kyaw.

Analysis and interpretation of data: G. C. Koh, H. M. Hoenig.

Drafting of manuscript: A. S. Narayanan.

Critical revision of manuscript for important intellectual content:

K. K. Tan, G. C. Koh, H. M. Hoenig.

Technical support: K. K. H. Kyaw.

Study supervision: K. K. Tan, H. M. Hoenig.

Financial Disclosures: The authors have declared that no competing interests exist.

Funding/Support: This material was based on work supported by an Economic Development Board Singapore-National Instruments Singapore Pte Ltd Industrial postgraduate program grant.

\section{REFERENCES}

1. Brienza DM, McCue M. Introduction to telerehabilitation. In: Kumar S, Cohn ER, editors. Telerehabilitation. London (UK): Springer-Verlag; 2013. p. 1-11.

2. Schein RM, Schmeler MR, Holm MB, Saptono A, Brienza DM. Telerehabilitation wheeled mobility and seating assessments compared with in person. Arch Phys Med Rehabil. 2010;91(6):874-78. [PMID:20510977] http://dx.doi.org/10.1016/j.apmr.2010.01.017

3. Sanou B. The world in 2013. ICT facts and figures 2013 [Internet]. Geneva (Switzerland): International Telecommunication Union; 2013 Feb [cited 2014 May 8]. Available from: http://www.itu.int/en/ITU-D/Statistics/Documents/ facts/ICTFactsFigures2013-e.pdf

4. Facts and figures [Internet]. Singapore: Info-communications Development Authority of Singapore; 2014 [cited 2014 May 8]. Available from: http://www.ida.gov.sg/Infocomm-Landscape/Facts-and-Figures

5. Price and performance of residential broadband service plans in Singapore [Internet]. Singapore: Info-communications Development Authority of Singapore; 2014 [cited 2014 May 8]. Available from: http://www.ida.gov.sg/applications/rbs/ chart.html

6. Olabi A. Fine motor activities. Houston (TX): Strategic Book Publishing; 2012.
7. Huetting C, Pyfer J, Auxter D. Gross motor activities for young children with special needs: A supplement to Auxter/Pyfer/Huettig principles and methods of adapted physical education and recreation. New York (NY): McGrawHill Higher Education; 2005.

8. Hoenig H, Tate L, Dumbleton S, Montgomery C, Morgan $\mathrm{M}$, Landerman LR, Caves K. A quality assurance study on the accuracy of measuring physical function under current conditions for use of clinical video telehealth. Arch Phys Med Rehabil. 2013;94(5):998-1002. [PMID:23337425] http://dx.doi.org/10.1016/j.apmr.2013.01.009

9. Arun SN, Tan KK. Video conferencing solution for medical applications in low-bandwidth networks. Proceedings of Australasian Telecommunication Networks and Applications Conference; 2013 Nov 20-22; Christchurch, New Zealand. 2013. p. 195-200.

10. Finkelstein J, Lapshin O, Castro H, Cha E, Provance PG. Home-based physical telerehabilitation in patients with multiple sclerosis: A pilot study. J Rehabil Res Dev. 2008; 45(9):1361-73. [PMID:19319760] http://dx.doi.org/10.1682/JRRD.2008.01.0001

11. Dick PT, Filler R, Pavan A. Participant satisfaction and comfort with multidisciplinary pediatric telemedicine consultations. J Pediatr Surg. 1999;34(1):137-42.

[PMID:10022159] http://dx.doi.org/10.1016/S0022-3468(99)90244-0

12. Rintala DH, Krouskop TA, Wright JV, Garber SL, Frnka J, Henson HK, Itani KM, Gaddis W, Matamoros R, Monga TN. Telerehabilitation for veterans with a lower-limb amputation or ulcer: Technical acceptability of data. J Rehabil Res Dev. 2004;41(3b):481-90. [PMID:15543466] http://dx.doi.org/10.1682/JRRD.2004.03.0481

13. Sanford JA, Hoenig, H. Comparison of in-home vs. telerehabilitation. Technol Aging. 2008;21:146-53.

14. Schein RM, Schmeler MR, Holm MB, Pramuka M, Saptono A, Brienza DM. Telerehabilitation assessment using the Functioning Everyday with a Wheelchair-Capacity instrument. J Rehabil Res Dev. 2011;48(2):115-24.

[PMID:21480086] http://dx.doi.org/10.1682/JRRD.2010.03.0039

15. Sanford JA, Griffiths PC, Richardson P, Hargraves K, Butterfield T, Hoenig $H$. The effects of in-home rehabilitation on task self-efficacy in mobility-impaired adults: A randomized clinical trial. J Am Geriatr Soc. 2006;54(11): 1641-48. [PMID:17087689] http://dx.doi.org/10.1111/j.1532-5415.2006.00913.x

16. Truter P, Russell T, Fary R. The validity of physical therapy assessment of low back pain via telerehabilitation in a clinical setting. Telemed J E Health. 2014;20(2):161-67. [PMID:24283249] http://dx.doi.org/10.1089/tmj.2013.0088 
17. Parmanto B, Saptono A, Pramana G, Pulantara W, Schein RM, Schmeler MR, McCue MP, Brienza DM. VISYTER: Versatile and integrated system for telerehabilitation. Telemed J E Health. 2010;16(9):939-44. [PMID:21034239] http://dx.doi.org/10.1089/tmj.2010.0033

18. Panayides A, Eleftheriou I, Pantziaris M. Open-source telemedicine platform for wireless medical video communication. Int J Telemed Appl. 2013;2013:457491. [PMID:23573082] http://dx.doi.org/10.1155/2013/457491

19. Chiu D, Jain R. Analysis of the increase and decrease algorithms for congestion avoidance in computer networks. Comput Netw ISDN Syst. 1989;17:1-14. http://dx.doi.org/10.1016/0169-7552(89)90019-6

20. Tan KK, Narayanan AS, Koh HC, Hoenig H, Caves K. Extraction of spatial information for low bandwidth telerehabilitation applications. J Rehabil Res Dev. 2014;51(5): 825-40. [PMID:25509058]
21. NetLimiter [Internet]. [cited 2014 May 8]. Available from: http://www.netlimiter.com/

Submitted for publication February 20, 2014. Accepted in revised form July 21, 2014.

This article and any supplementary material should be cited as follows:

Tan KK, Narayanan AS, Koh GC, Kyaw KK, Hoenig HM. Development of telerehabilitation application with designated consultation categories. J Rehabil Res Dev. 2014;51(9):1383-96.

http://dx.doi.org/10.1682/JRRD.2014.02.0052

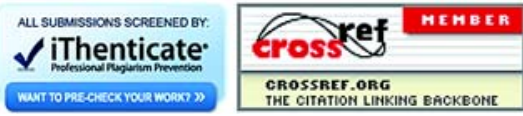

